\title{
A topological formulation for exotic quantum holonomy
}

\author{
A. Tanaka ${ }^{1}$, T.Cheon ${ }^{2}$ \\ ${ }^{1}$ Department of Physics, Tokyo Metropolitan University, \\ Hachioji, Tokyo 192-0397, Japan \\ ${ }^{2}$ Laboratory of Physics, Kochi University of Technology, \\ Tosa Yamada, Kochi 782-8502, Japan \\ tanaka-atushi@tmu.ac.jp
}

PACS 03.65.-w,03.65.Vf,02.40.Pc

DOI 10.17586/2220-8054-2015-6-6-786-792

\begin{abstract}
An adiabatic change of parameters along a closed path may interchange the (quasi-)eigenenergies and eigenspaces of a closed quantum system. Such discrepancies, induced by adiabatic cycles are referred to as the exotic quantum holonomy, which is an extension of the geometric phase. "Small" adiabatic cycles induce no change on eigenspaces, whereas some "large" adiabatic cycles interchange eigenspaces. We explain the topological formulation for the eigenspace anholonomy, where the homotopy equivalence precisely distinguishes the larger cycles from smaller ones. An application to two level systems is explained. We also examine the cycles that involve the adiabatic evolution across an exact crossing, and the diabatic evolution across an avoided crossing. The latter is a nonadiabatic example of the exotic quantum holonomy.
\end{abstract}

Keywords: exotic quantum holonomy, homotopy, disclination.

Received: 1 November 2015

\section{Introduction}

An adiabatic quasi-static cycle may induce a nontrivial change on a closed quantum system. A well-known example is the geometric phase factor $[1,2]$, which is also called as the quantum holonomy because of its geometrical interpretation $[3,4]$.

Recently, it has been recognized that the geometric phase has exotic relatives, where (quasi-)eigenenergies and eigenspaces of stationary states exhibit nontrivial change as a result of an adiabatic cycle. Namely, eigenenergies and eigenspaces may be interchanged by adiabatic cycles.

The exotic quantum holonomy has been found in various physical systems: a particle confined in a one-dimensional box with a generalized pointlike potential [5], a quantum map under a rank-1 perturbation [6], the Lieb-Liniger model [7], and a quantum graph [8]. Other examples are reported in the references cited in Ref. [9].

In the following, we will briefly explain the topological formulation for the exotic quantum holonomy [9], which may be considered as a counterpart to the geometrical formulation for the geometric phase factor $[3,10]$. Using the homotopic classification, we discuss "large" cycles that exhibit exotic quantum holonomy in Hamiltonian systems that involve the exact level crossing and avoided crossing. 


\section{Topological formulation}

The quantum holonomies including both the geometric phase and the eigenspace anholonomy, are discrepancies induced by an adiabatic cycle, i.e., a closed path in an adiabatic parameter space $\mathcal{M}$. A lifting of the adiabatic cycle is helpful to characterize the quantum holonomies.

As for the geometric phase, we define a lift of the adiabatic path as the trajectory of the state vector, which satisfies the adiabatic time-dependent Schrödinger equation with an adiabatic initial state. We assume that the dynamical phase is excluded from the lift.

The lift of an adiabatic cycle $C$ induces a mapping, which is denoted by $\phi_{C}$, from the initial adiabatic state to the final adiabatic state. $\phi_{C}$ puts a geometric phase factor to the initial state vector. In terms of differential geometry, $\phi_{C}$ is an element of the holonomy group. This fact allows us to thoroughly investigate the geometric phase with the help of differential geometry [3].

(a)

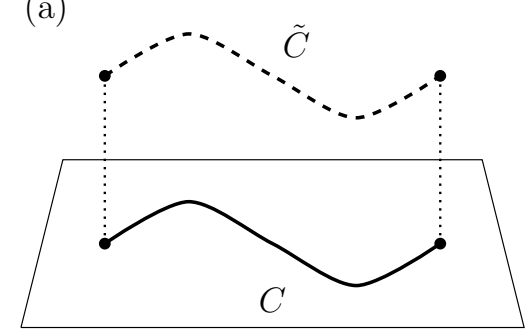

(b)

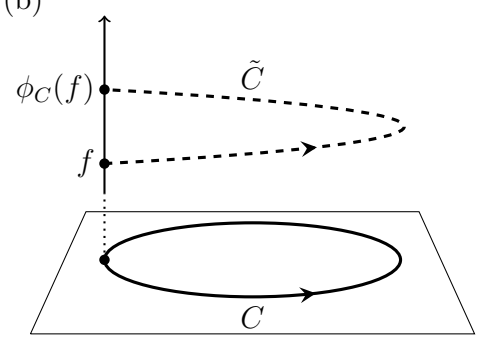

FIG. 1. Lifting structure behind quantum holonomies. (a) Lift $\tilde{C}$ (dashed line) of a path $C$ (bold line) in the adiabatic parameter space $\mathcal{M}$. A lift is the trajectory of an eigenobject under the adiabatic time evolution with an adiabatic initial condition. The dotted lines represent the projection $\pi$ from the lifted space to $\mathcal{M}$. As for the case of the geometric phase, the lift is the trajectory of the state vector excluding the dynamical phase. On the other hand, the lift for the exotic quantum holonomy is the trajectory of $p$ (Eq. (1)). (b) Lift of the closed path (i.e., adiabatic cycle) for a given initial eigenobject $f$. We denote the final point of the lift by $\phi_{C}(f)$. The mapping $\phi_{C}$ describes the discrepancy of the eigenobject induced by the cycle $C$

We carry over the concept of lifting to the exotic quantum holonomy. Instead of the adiabatic state vector for the geometric phase, we employ an ordered set of eigenprojectors

$$
p \equiv\left(\hat{P}_{1}, \hat{P}_{2}, \ldots\right)
$$

where $\hat{P}_{j}$ is $j$-th eigenprojector under a given value of the adiabatic parameter. We denote $p$-space by $\mathcal{P}$.

We explain two kinds of adiabatic parameter space $\mathcal{M}$, which is located at the bottom of the lifting structure. One is a $c$-number parameter space. Alternatively, it is useful to introduce a canonical "adiabatic parameter space", whose point is a set of projectors:

$$
b \equiv\left\{\hat{P}_{1}, \hat{P}_{2}, \ldots\right\}
$$

where the order of the projectors are disregarded. $b$-space is a counterpart of the projective Hilbert space in the Aharonov-Anandan theory of the geometric phase [10].

We explain the lifting of an adiabatic cycle $C$ in $\mathcal{M}$ to $\mathcal{P}$, and the corresponding $\phi_{C}$. For a given adiabatic path, the adiabatic Schrödinger equation induces the adiabatic 
time evolution of $p$. The lifted path is the trajectory of $p$, and naturally induces $\phi_{C}$. The mapping of $\phi_{C}$ between the initial and final $p$ is essentially a permutation of $\hat{P}_{j}$ 's. For example, if $C$ does not induces the exotic quantum holonomy, $\phi_{C}$ is equivalent with the identical permutation. On the other hand, $\phi_{C}$ may be a non-identical permutation, which describes the interchange of the eigenspaces, induced by $C$.

Here, our task is to characterize $\phi_{C}$ completely. We utilize the fact that there is a covering map $\pi: \mathcal{P} \rightarrow \mathcal{M}$, where $\pi$ is the projection that satisfies the axiom of the covering projection (not shown here). We remark that the covering space is a fiber bundle with a discrete structure group due to the discreteness of $p$ for a given value of the adiabatic parameter.

The covering map structure allows us to investigate the exotic quantum holonomy with the help of topology. First, $\phi_{C}$ and $\phi_{C^{\prime}}$ are the same if $C$ and $C^{\prime}$ are homotopic, where we say that a cycle $C$ is homotopic to another cycle $C^{\prime}$ if $C$ can be smoothly deformed to $C^{\prime}$ with the initial and final points remaining unchanged. Hence, we may denote the mapping $\phi_{C}$ as $\phi_{[C]}$, where $[C]$ is the class of paths that are homotopic to $C$.

Next, we need to enumerate all possible $[C]$ 's in the adiabatic parameter space $\mathcal{M}$. This is equivalent to finding $\pi_{1}(\mathcal{M})=\{[C] \mid C$ is a closed path in $\mathcal{M}\}$, which is called the first fundamental group of $\mathcal{M}$.

Hence, it suffices to enumerate $\phi_{[C]}$ for all $[C] \in \pi_{1}(M)$. When $\mathcal{P}$ is contractable to a point (i.e., there is no "hole"), there is a one-to-one correspondence between $\pi_{1}(\mathcal{M})$ and $\phi_{[C]}$, i.e.:

$$
\left\{\phi_{[C]}\right\}_{[C] \in \pi_{1}(\mathcal{M})} \simeq \pi_{1}(\mathcal{M}) .
$$

In other words, if $C$ is not homotopic to $C^{\prime}$, two permutations $\phi_{[C]}$ and $\phi_{\left[C^{\prime}\right]}$ are different. The extension of Eq. (3) to an arbitrary $\mathcal{P}$ is shown in Ref. [9].

\section{Analysis of two level systems}

We examine the exotic quantum holonomy in two level systems. For a while, we suppose the system has no spectral degeneracy. Hence, the Hamiltonian $\hat{H}$ has the following spectral decomposition:

$$
\hat{H}=E_{1} \hat{P}_{1}+E_{2} \hat{P}_{2} .
$$

where $E_{1}$ and $E_{2}$ are eigenvalues of $H$. In two level systems, the eigenprojections $\hat{P}_{1}$ and $\hat{P}_{2}$ may be specified by a normalized 3-vector $\boldsymbol{a}$, which is called as the Bloch vector, as

$$
\hat{P}_{1}=\frac{1}{2}(1+\boldsymbol{a} \cdot \hat{\boldsymbol{\sigma}}), \quad \hat{P}_{2}=\frac{1}{2}(1-\boldsymbol{a} \cdot \hat{\boldsymbol{\sigma}}) .
$$

Non-degenerate periodically driven systems can be examined in the same manner once we replace $\hat{H}$ with a Floquet operator.

We explain that $p$ and $b$ have a simple geometrical interpretation in two level systems (see, Fig. 2 (a)). First, $p$ is equivalent to $\boldsymbol{a}$, a point in a sphere $S^{2}$, as is seen from Eq. (5). Second, $b$ is equivalent to the director (headless vector) $\boldsymbol{n}$ [11], which correspond to a point in the real projective plane $\mathbb{R} P^{2}$. This is because both $\boldsymbol{a}$ and $-\boldsymbol{a}$ correspond to the same value of $b$, and the identification of antipodal point on the sphere leads to $\mathbb{R} P^{2}$.

Once we employ $\boldsymbol{n}$ as the adiabatic parameter space, the topological formulation provides an intuitive interpretation of the exotic quantum holonomy. The lifts of a "small" cycle in $\boldsymbol{n}$-space are closed in $\boldsymbol{a}$-space, i.e., the sphere (Fig. 2 (b)). Conversely, the lifts of a "large" cycle in $\boldsymbol{n}$-space can be open in $\boldsymbol{a}$-space. The exotic quantum holonomy reflects such a discrepancy between the trajectories of $\boldsymbol{n}$ and $\boldsymbol{a}$. 
A complete way to distinguish the smaller and larger cycles is provided by the homotopy equivalence. In $\boldsymbol{n}$-space, i.e., $\mathbb{R} P^{2}$, there are only two classes of cycles, i.e., $\pi_{1}\left(\mathbb{R} P^{2}\right)=\{[e],[\gamma]\}$, where $e$ is a "small" cycle that is homotopic to a point (i.e., the zero-length closed path), and a "large" cycle $\gamma$ is not homotopic to $e$ (Fig. 2 (b)).

Now Eq. (3) is applicable to classify $\phi_{[C]}$ completely, since $\boldsymbol{a}$-space, i.e. $S^{2}$, has no hole. Namely, $\phi_{[e]}$ and $\phi_{[\gamma]}$ correspond to different permutations, the identical and the cyclic permutations of two items, respectively (see, Fig. 2 (b)).

(a)

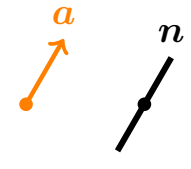

(b)

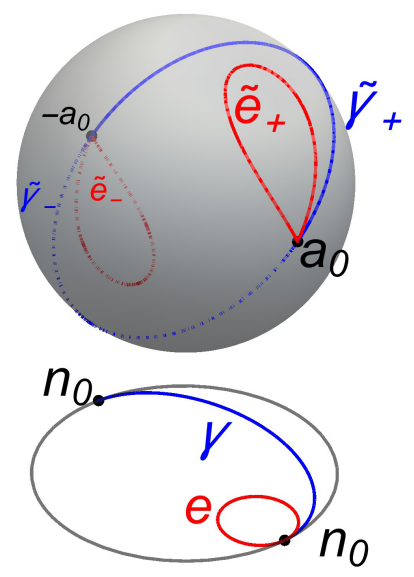

Fig. 2. (a) Bloch vector $\boldsymbol{a}$ and director $\boldsymbol{n}$ (schematic). (b) Two homotopically inequivalent cycles $e$ and $\gamma$ in $\mathbb{R} P^{2}$ (bottom), and their lifts to $S^{2}$ (upper). The initial point of the cycles is denoted as $\boldsymbol{n}_{0}$. The lifts of the cycles with initial point $\pm \boldsymbol{a}_{0}$ are $\tilde{e}_{ \pm}$and $\tilde{\gamma}_{ \pm}$. Since the former are closed in $S^{2}, \phi_{[e]}$ correspond to the identical permutation of eigenspaces. On the other hand, the latter are open so that $\phi_{[\gamma]}$ corresponds to the cyclic permutation of the two eigenspaces, i.e., $\gamma$ induces the exotic quantum holonomy

\section{Examples of nontrivial cycle $\gamma$}

We explain how we realize the adiabatic cycles $\gamma$, which induces the cyclic permutation of eigenspaces as well as (quasi-)eigenenergies in non-degenerate two level systems.

The first example is a family of a quantum map under a rank-1 perturbation, which is described by a periodically-kicked Hamiltonian $\hat{H}(\lambda, t)=\hat{H}_{0}+\lambda|v\rangle\langle v| \sum_{n=-\infty}^{\infty} \delta(t-n)$, where $|v\rangle$ is a normalized vector. We introduce a Floquet operator, which describes the time evolution during a unit time interval,

$$
\hat{U}(\lambda)=e^{-i \hat{H}_{0}} e^{-i \lambda|v\rangle\langle v|}
$$

The stationary state of this system for a given value of $\lambda$ is described by an eigenvector of $\hat{U}(\lambda)$, and the time evolution induced by an adiabatic variation of $\lambda$ is essentially governed by the parametric evolution of eigenvectors of $\hat{U}(\lambda)[12]$. Since $\hat{U}(\lambda)$ has a period $2 \pi$ as a function of $\lambda$, the trajectory of $\boldsymbol{n}$ induced by $\hat{U}(\lambda)$ for $0 \leqslant \lambda \leqslant 2 \pi$ makes a closed path in $\mathbb{R} P^{2}$. Nevertheless, the lift of the closed path to $S^{2}$ is generically open to exhibit the nonidentical permutation of eigenprojectors, as these closed paths are homotopic to $\gamma$ (Fig. 3). See, Refs. [6,9] for details. 


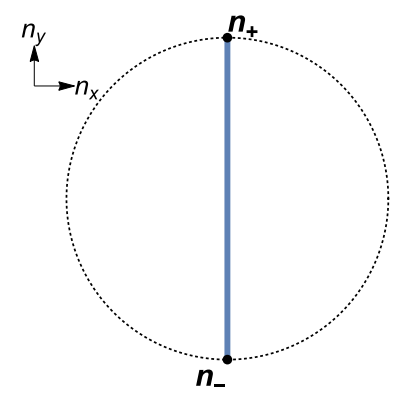

Fig. 3. Trajectories of the director $\boldsymbol{n}$. We depict the trajectories in $n_{x}-n_{y}$ plane. The first example is the quantum map (6) with $\hat{H}_{0}=\frac{\pi}{2} \hat{\sigma}_{y}$ and $|v\rangle\langle v|=$ $\left(1+\hat{\sigma}_{x}\right) / 2$, which implies $\boldsymbol{a}=\boldsymbol{e}_{y} \cos (\lambda / 2)-\boldsymbol{e}_{z} \sin (\lambda / 2)$, whose director moves along $n_{y}$-axis. Note that the initial and end points $\boldsymbol{n}_{ \pm}$are same in $\mathbb{R} P^{2}$. Hence the trajectory is closed, and homotopic to $\gamma$. The Bloch vector of the second example (7) is shown in Eq. (8). The trajectory of the director coincides with the one of the first example, and agrees with the one for the third nonadiabatic example

Second, we examine the adiabatic cycles that involve a level crossing [13] using the following Hamiltonian

$$
\hat{H}(\lambda) \equiv \frac{1}{4}\left[(1+\cos \lambda) \hat{\sigma}_{y}+(\sin \lambda) \hat{\sigma}_{z}\right]
$$

which is periodic in $\lambda$ with a period $2 \pi$, and degenerates at $\lambda=\pi$. We introduce a Bloch vector

$$
\boldsymbol{a} \equiv \cos (\lambda / 2) \boldsymbol{e}_{y}+\sin (\lambda / 2) \boldsymbol{e}_{z}
$$

for Eq. (7), which leads to the spectrum decomposition (4) with the eigenprojectors (5), $E_{1}=$ $\frac{1}{2} \cos (\lambda / 2)$ and $E_{2}=-\frac{1}{2} \cos (\lambda / 2)$ (Fig. 4). We note that the Bloch vector $\boldsymbol{a}$ smoothly depends on $\lambda$ even in the vicinity of the crossing point $\lambda=\pi$. Hence, the adiabatic time evolution follows the corresponding parametric evolution of eigenprojectors (5) [14]. Although $\hat{H}(\lambda)$ is periodic in $\lambda$ with the period $2 \pi$, the eigenenergies, as well as the eigenprojectors, are not, which implies the presence of the exotic quantum holonomy. Hence, the trajectory of the director for $0 \leqslant \lambda \leqslant 2 \pi$ is closed, and is homotopic to $\gamma$ (see, Fig. 3).

Third, we examine the second case under a generic small perturbation, which generically breaks the spectral degeneracy. For example, we consider:

$$
\hat{H}_{\epsilon}(\lambda) \equiv \hat{H}(\lambda)+\frac{1}{2} \epsilon \sigma_{x}
$$

The level crossing point becomes an avoided crossing point due to the perturbation (Fig. 4). Hence, the exotic quantum holonomy do not occur in the adiabatic limit. However, the use of the diabatic process across the avoided crossing recovers the exotic quantum holonomy [13]. Along the closed path $0 \leqslant \lambda \leqslant 2 \pi$ involving the diabatic process, the trajectory induced by the time evolution of the state projector, which is initially an eigenprojector of $\hat{H}(0)$, mimics the parametric evolution of the corresponding eigenprojector of the unperturbed system $\hat{H}(\lambda)$. In this sense, the diabatic cycle plays the role of nontrivial cycle $\gamma$. We remark that this is a nonadiabatic example of the exotic quantum holonomy. 


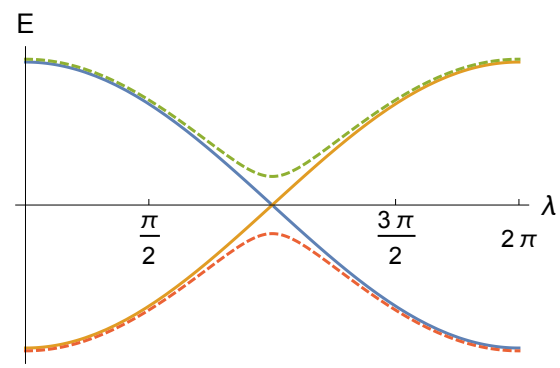

FIG. 4. Eigenenergies of the level crossing Hamiltonian $\hat{H}(\lambda)$ (Eq. (7)) (thick lines) and the perturbed Hamiltonian $\hat{H}_{\epsilon}(\lambda)$ (Eq. (9)) (dashed lines). Since the eigenenergies of $\hat{H}(\lambda)$ crosses at $\lambda=\pi$, the adiabatic cycle $0 \leqslant \lambda \leqslant 2 \pi$ interchanges the eigenspaces at $\lambda=0$. The adiabatic cycle for $\hat{H}_{\epsilon}(\lambda)$ do not induce the exotic quantum holonomy, due to the presence of the avoided crossing around $\lambda=\pi$. However, if $\lambda$ is moved quickly around $\lambda=\pi$, the system follows the diabatic evolution. Such a diabatic cycle induces the exotic quantum holonomy along the nonadiabatic time evolution [13]

\section{Summary}

We briefly explained the topological formulation of the exotic quantum holonomy with an emphasis to its geometrical character in two level systems. An application to Hamiltonian systems with an exact or avoided crossing is explained.

\section{Acknowledgements}

This research was supported by the Japan Ministry of Education, Culture, Sports, Science and Technology under the Grant number 15K05216.

\section{References}

[1] M. V. Berry. Quantal phase factors accompanying adiabatic changes. Proc. R. Soc. London A, 1984, 392 P. 45.

[2] A. Shapere, F. Wilczek (Eds.). Geometric phases in physics. World Scientific, Singapore, 1989.

[3] B. Simon, Holonomy, the Quantum Adiabatic Theorem, and Berry's Phase. Phys. Rev. Lett., 1983, 51, P. 2167.

[4] A. Bohm, A. Mostafazadeh, H. Koizumi, Q. Niu, Z. Zwanziger. The Geometric Phase in Quantum Systems, Springer, Berlin, 2003; D. Chruściński, A. Jamiołkowski. Geometric Phases in Classical and Quantum Mechanics. Birkhäuser, Boston, 2004.

[5] T. Cheon. Double spiral energy surface in one-dimensional quantum mechanics of generalized pointlike potentials. Phys. Lett. A, 1998, 248, P. 285.

[6] A. Tanaka, M. Miyamoto. Quasienergy anholonomy and its application to adiabatic quantum state manipulation. Phys. Rev. Lett., 2007, 98, P. 160407.

[7] N. Yonezawa, A. Tanaka, T. Cheon. Quantum holonomy in the Lieb-Liniger model. Phys. Rev. A, 2013, 87, P. 062113.

[8] T. Cheon, A. Tanaka, O. Turek. Examples of quantum holonomy with topology changes. Acta Polytechnica, 2013, 53, P. 410.

[9] A. Tanaka, T. Cheon. Bloch vector, disclination and exotic quantum holonomy. Phys. Lett. A, 2015, 379, P. 1693.

[10] Y. Aharonov, J. Anandan. Phase change during a cyclic quantum evolution. Phys. Rev. Lett., 1987, 58, P. 1593.

[11] P. G. de Gennes, J. Prost. The Physics of Liquid Crystals, 2-nd Edition, Oxford University Press, Oxford, 1993, Ch. 1; N. D. Mermin. The topological theory of defects in ordered media. Rev. Mod. Phys., 1979, 51, P. 591. 
[12] A. Dranov, J. Kellendonk, R. Seiler. Discrete time adiabatic theorems for quantum mechanical systems. J. Math. Phys., 1998, 39, P. 1340. A. Tanaka. Adiabatic theorem for discrete time evolution. J. Phys. Soc. Japan, 2011, 80, P. 125002.

[13] T. Cheon, A. Tanaka, S. W. Kim. Exotic quantum holonomy in Hamiltonian systems. Phys. Lett. A, 2009, 374, P. 144.

[14] T. Kato. On the Adiabatic Theorem of Qunatum Mechanics. J. Phys. Soc. Japan, 1950, 5, P. 435. 